\title{
Entropy formula in Einstein-Maxwell-dilaton theory and its validity for black strings
}

\author{
Mohammad Reza Setare* \\ Department of Science, Campus of Bijar, University of Kurdistan, 98 Bijar, Iran \\ Hamed Adami ${ }^{\dagger}$ \\ Research Institute for Astronomy and Astrophysics of Maragha (RIAAM), \\ P.O. Box 55134-441, Maragha, Iran
}

(Received 25 May 2018; published 10 October 2018)

\begin{abstract}
We consider the near-horizon fall-off conditions of stationary black holes in Einstein-Maxwell-Dilaton theory and find a conserved charge conjugate to the symmetry generator that preserves these conditions. Subsequently, we find supertranslation, superrotation, and multiple-charge modes and calculate them for two spacial examples: a typical static dilaton black hole and a charged rotating black string. In EinsteinMaxwell-Dilaton theory, the supertranslation double-zero-mode charge $\mathcal{T}_{(0,0)}$ is not equal to the product of the black hole entropy and the Hawking temperature. This may be seen as a problem, but it is not. There is a $U(1)$ gauge freedom, and we use gauge fixing to fix the problem. We show that the new entropy formula $4 \pi \hat{J}_{0}^{+} \hat{J}_{0}^{-}$, proposed by Gonzalez et al. [EPJ Web Conf. 168, 01009 (2018)], is valid for black strings as well as black holes.
\end{abstract}

DOI: 10.1103/PhysRevD.98.084015

\section{INTRODUCTION}

The Einstein-Maxwell-dilaton (EMD) theory originating from a low-energy limit of string theory allows for black holes that have mass, rotation, charge, and scalar hair [1]. The uniqueness of static, asymptotically flat spacetimes with nondegenerate black holes of EMD theory was investigated in Ref. [2]. The dilaton field can change the asymptotic behavior of the solutions to be neither asymptotically flat nor anti-de Sitter (A)dS. Rotating solutions of EMD theory with a Liouville-type potential in four and $(n+1)$ dimensions with flat horizons have been studied, respectively, in Refs. [3,4] and [4]. These solutions describe charged rotating dilaton black strings/branes.

In this paper we would like to provide the first nontrivial evidence for the universality of the entropy formula $4 \pi J_{0}^{+} J_{0}^{-}$in EMD theory in four dimensions. Recently, the above entropy formula emerged in the near-horizon description of nonextremal Kerr black holes in four dimensions [5]. In a previous paper [6] we showed that this entropy formula gives the correct results for Kerr-

\footnotetext{
*rezakord@ipm.ir

†hamed.adami@yahoo.com
}

Published by the American Physical Society under the terms of the Creative Commons Attribution 4.0 International license. Further distribution of this work must maintain attribution to the author(s) and the published article's title, journal citation, and DOI. Funded by SCOAP .
Newman (A)dS black holes. In order to investigate the universality of the above entropy formula, here we study the near-horizon fall-off conditions of stationary black holes in EMD theory. We show that the above new entropy formula is not only valid for black hole solutions, but that it also works correctly for black string solutions. For this propose, we use the covariant phase space method for obtaining conserved charges in EMD theory. Then, we study the near-horizon behavior of a stationary black hole in EMD theory. We find a conserved charge conjugate to the symmetry generator that preserves near-horizon fall-off conditions. After that, we obtain supertranslation, superrotation, and multiple-charge modes.

\section{CONSERVED CHARGES IN EINSTEIN- MAXWELL-DILATON THEORY}

Let us consider EMD theory. The Lagrangian describing EMD theory is a functional of the metric $g_{\mu \nu}, U(1)$ gauge field $A_{\mu}$, and a real scalar field $\varphi$,

$L=\sqrt{-g}\left(\mathcal{R}-2 \Lambda-\frac{1}{2} \partial_{\mu} \varphi \partial^{\mu} \varphi-\frac{1}{4} \mathcal{N}(\varphi) F_{\mu \nu} F^{\mu \nu}-\mathcal{V}(\varphi)\right)$

where $\mathcal{R}, F_{\mu \nu}=\partial_{\mu} A_{\nu}-\partial_{\nu} A_{\mu}$, and $\Lambda$ are, respectively, the Ricci scalar, electromagnetic field strength, and cosmological constant. 
Now, we briefly review the approach of the covariant phase space method for obtaining conserved charges in EMD theory. To do this, we follow Refs. [7-11]. The firstorder variation of the Lagrangian (1) is

$$
\delta L[\Phi]=E_{\Phi}[\Phi] \delta \Phi+\partial_{\mu} \Theta^{\mu}[\Phi, \delta \Phi],
$$

where $\Phi=\left\{g_{\mu \nu}, A_{\mu}, \varphi\right\}$ denotes the collection of dynamical fields, $E_{\Phi}$ have dual indices with $\Phi$, and a sum on $\Phi$ is explicitly assumed. In Eq. (2),

$$
\Theta^{\mu}[\Phi, \delta \Phi]=\sqrt{-g}\left\{2 \nabla^{[\alpha}\left(g^{\mu] \beta} \delta g_{\alpha \beta}\right)-\partial^{\mu} \varphi \delta \varphi-\mathcal{N} F^{\mu \nu} \delta A_{\nu}\right\}
$$

is the surface term and we refer to this as the symplectic potential. Also, $E_{\Phi}=0$ gives us the field equations

$$
\begin{gathered}
G^{\mu \nu}+\Lambda g^{\mu \nu}=T_{(A)}^{\mu \nu}+T_{(\varphi)}^{\mu \nu}, \\
\nabla_{\nu}\left(\mathcal{N} F^{\nu \mu}\right)=0, \\
\square \varphi-\mathcal{V}^{\prime}-\frac{1}{4} \mathcal{N}^{\prime} F_{\alpha \beta} F^{\alpha \beta}=0,
\end{gathered}
$$

where $G^{\mu \nu}$ is the Einstein tensor and

$$
\begin{gathered}
T_{(A)}^{\mu \nu}=\frac{1}{2} \mathcal{N}\left(F^{\mu \alpha} F^{\nu}{ }_{\alpha}-\frac{1}{4} g^{\mu \nu} F^{\alpha \beta} F_{\alpha \beta}\right), \\
T_{(\varphi)}^{\mu \nu}=\frac{1}{2} \partial^{\mu} \varphi \partial^{\nu} \varphi-\frac{1}{2} g^{\mu \nu}\left(\frac{1}{2} \partial_{\alpha} \varphi \partial^{\alpha} \varphi+\mathcal{V}\right)
\end{gathered}
$$

are the contributions of the electromagnetic field and scalar field in the energy-momentum tensor, respectively. Here, the prime symbol denotes differentiation with respect to $\varphi$.

Now we consider two arbitrary variations $\delta_{1}$ and $\delta_{2}$. Suppose that these two variations do not commute, $\delta_{1} \delta_{2} \neq \delta_{2} \delta_{1}$. By varying Eq. (2), we find the second-order variation of the Lagrangian,

$\delta_{1} \delta_{2} L[\Phi]=\delta_{1} E_{\Phi}[\Phi] \delta_{2} \Phi+E_{\Phi}[\Phi] \delta_{1} \delta_{2} \Phi+\partial_{\mu} \delta_{1} \Theta^{\mu}\left[\Phi, \delta_{2} \Phi\right]$.

Similarly, one can write

$\delta_{2} \delta_{1} L[\Phi]=\delta_{2} E_{\Phi}[\Phi] \delta_{1} \Phi+E_{\Phi}[\Phi] \delta_{2} \delta_{1} \Phi+\partial_{\mu} \delta_{2} \Theta^{\mu}\left[\Phi, \delta_{1} \Phi\right]$.

By subtracting Eq. (10) from Eq. (9), we have

$$
\begin{aligned}
\delta_{[1,2]} L[\Phi]= & \delta_{1} E_{\Phi}[\Phi] \delta_{2} \Phi-\delta_{2} E_{\Phi}[\Phi] \delta_{1} \Phi+E_{\Phi}[\Phi] \delta_{[1,2]} \Phi \\
& +\partial_{\mu}\left(\delta_{1} \Theta^{\mu}\left[\Phi, \delta_{2} \Phi\right]-\delta_{2} \Theta^{\mu}\left[\Phi, \delta_{1} \Phi\right]\right),
\end{aligned}
$$

where $\delta_{[1,2]}=\delta_{1} \delta_{2}-\delta_{2} \delta_{1}$ is the commutator of the two variations $\delta_{1}$ and $\delta_{2}$. By using Eq. (2) and replacing $\delta \rightarrow \delta_{[1,2]}$, we can write Eq. (11) as

$\partial_{\mu} \omega_{\mathrm{LW}}^{\mu}\left[\Phi ; \delta_{1} \Phi, \delta_{2} \Phi\right]=-\frac{1}{16 \pi}\left(\delta_{1} E_{\Phi}[\Phi] \delta_{2} \Phi-\delta_{2} E_{\Phi}[\Phi] \delta_{1} \Phi\right)$,

where

$$
\begin{aligned}
& \omega_{\mathrm{LW}}^{\mu}\left[\Phi ; \delta_{1} \Phi, \delta_{2} \Phi\right] \\
& \quad=\frac{1}{16 \pi}\left(\delta_{1} \Theta^{\mu}\left[\Phi, \delta_{2} \Phi\right]-\delta_{2} \Theta^{\mu}\left[\Phi, \delta_{1} \Phi\right]-\Theta^{\mu}\left[\Phi, \delta_{[1,2]} \Phi\right]\right)
\end{aligned}
$$

is the Lee-Wald symplectic current. Since the symplectic potential is linear in $\delta \Phi$, the terms containing $\delta_{1} \delta_{2} \Phi$, $\delta_{2} \delta_{1} \Phi$, and $\delta_{[1,2]} \Phi$ eliminate each other and $\omega_{\mathrm{LW}}^{\mu}$ is a skew-symmetric bilinear in $\delta_{1} \Phi$ and $\delta_{2} \Phi$. The Lee-Wald symplectic current is conserved when the equations of motion and linearized equations of motion are satisfied. In other words, if $\Phi$ is a solution of $E_{\Phi}=0$ and $\delta_{1} \Phi$ and $\delta_{2} \Phi$ are solutions of $\delta E_{\Phi}=0$, then the Lee-Wald symplectic current is conserved,

$$
\partial_{\mu} \omega_{\mathrm{LW}}^{\mu}\left[\Phi ; \delta_{1} \Phi, \delta_{2} \Phi\right] \simeq 0 .
$$

The sign $\simeq$ indicates that the equality is held on-shell. We can define the symplectic 2-form in solution space through the Lee-Wald symplectic current,

$$
\Omega_{\mathrm{LW}}\left[\Phi ; \delta_{1} \Phi, \delta_{2} \Phi\right]=\int_{\mathcal{C}} \omega_{\mathrm{LW}}^{\mu}\left[\Phi ; \delta_{1} \Phi, \delta_{2} \Phi\right] d^{3} x_{\mu},
$$

where $\mathcal{C}$ is a codimension- 1 spacelike surface. The solution phase space can be constructed by factoring out the degeneracy subspace of the configuration space (see Ref. [7] for a detailed discussion). Hence, $\Omega_{\mathrm{Lw}}$ will be a symplectic form in solution phase space and it is closed, skew-symmetric, and nondegenerate.

Suppose $\xi^{\mu}(x)$ and $\lambda(x)$ are the diffeomorphism and $U(1)$ gauge transformation generators, respectively. We can introduce a combined transformation so that $\chi=(\xi, \lambda)$ is the generator of such a transformation [6,12]. The changes in the metric, $U(1)$ gauge field, and scalar field induced by an infinitesimal transformation generated by $\chi$ are given by

$$
\begin{gathered}
\delta_{\chi} g_{\mu \nu}=\mathcal{L}_{\xi} g_{\mu \nu}, \\
\delta_{\chi} A_{\mu}=\mathcal{L}_{\xi} A_{\mu}+\partial_{\mu} \lambda, \\
\delta_{\chi} \varphi=\mathcal{L}_{\xi} \varphi,
\end{gathered}
$$

respectively. Here, $\mathcal{L}_{\xi}$ denotes the Lie derivative along the vector field $\xi$. Also, the change in the Lagrangian (1) induced by an infinitesimal transformation generated by $\chi$ is 


$$
\delta_{\chi} L[\Phi]=\mathcal{L}_{\xi} L[\Phi]=\partial_{\mu}\left(\xi^{\mu} L[\Phi]\right) .
$$

Since changes in the dynamical fields are linear in the generator $\chi$ and the change in the Lagrangian is a total derivative, then $\chi$ generates a local symmetry in solution phase space [7]. The generators of such a local symmetry in solution phase space are conserved charges. The charge perturbation conjugate to $\chi$ is defined as

$$
\delta Q_{\chi}=\Omega_{\mathrm{LW}}\left[\Phi ; \delta \Phi, \delta_{\chi} \Phi\right] .
$$

The algebra among conserved charges is

$$
\left\{Q_{\chi_{1}}, Q_{\chi_{2}}\right\}=Q_{\left[\chi_{1}, \chi_{2}\right]}+\tilde{\mathcal{C}}\left(\chi_{1}, \chi_{2}\right)
$$

where $\tilde{\mathcal{C}}\left(\chi_{1}, \chi_{2}\right)$ is the central extension term and the Dirac bracket is defined as

$$
\left\{Q_{\chi_{1}}, Q_{\chi_{2}}\right\}=\delta_{\chi_{2}} Q_{\chi_{1}} .
$$

Now, we want to find the explicit form of conserved charges in the EMD theory. To this end, we assume that the variation in Eq. (2) is induced by an infinitesimal transformation generated by $\chi$,

$$
\delta_{\chi} L[\Phi] \simeq \partial_{\mu} \Theta^{\mu}\left[\Phi, \delta_{\chi} \Phi\right] .
$$

Then, we can define an on-shell Noether current

$$
J_{\mathrm{N}}^{\mu}[\Phi ; \chi] \simeq \Theta^{\mu}\left[\Phi, \delta_{\chi} \Phi\right]-\xi^{\mu} L[\Phi],
$$

which is conserved on shell, i.e., $\partial_{\mu} J_{\mathrm{N}}^{\mu} \simeq 0$. Thus, there exists a second-rank tensor density of weight +1 ,

$K_{\mathrm{N}}^{\mu \nu}[\Phi ; \chi] \simeq-\sqrt{-g}\left\{2 \nabla^{[\mu} \xi^{\nu]}+\mathcal{N} F^{\mu \nu}\left(\lambda+A_{\alpha} \xi^{\alpha}\right)\right\}$,

so that $J_{\mathrm{N}}^{\mu} \simeq \partial_{\nu} K_{\mathrm{N}}^{\mu \nu}$. We refer to $K_{\mathrm{N}}^{\mu \nu}$ as the Noether potential. To find the explicit form of the symplectic current, we first take an arbitrary variation of Eq. [24],

$$
\partial_{\nu} \delta K_{\mathrm{N}}^{\mu \nu}[\Phi ; \chi] \simeq \delta \Theta^{\mu}\left[\Phi, \delta_{\chi} \Phi\right]-\delta\left(\xi^{\mu} L[\Phi]\right) .
$$

For generality, we assume that $\chi$ depends on the dynamical fields. On the other hand, the second variation of Eq. (1), induced by an infinitesimal transformation generated by $\chi$, is

$$
\delta_{\chi} \delta L[\Phi] \simeq \partial_{\mu} \delta_{\chi} \Theta^{\mu}[\Phi, \delta \Phi] .
$$

Since the commutator of an arbitrary variation and a variation induced by an infinitesimal transformation generated by $\chi$ is $\delta \delta_{\chi}-\delta_{\chi} \delta=\delta_{\delta \chi}$, Eq. (27) can be written as

$$
\delta \delta_{\chi} L[\Phi]-\delta_{\delta \chi} L[\Phi] \simeq \partial_{\mu} \delta_{\chi} \Theta^{\mu}[\Phi, \delta \Phi] .
$$

By substituting Eq. (23) into Eq. (28), we find the explicit form of the symplectic current as

$$
\omega_{\mathrm{LW}}^{\mu}\left[\Phi ; \delta \Phi, \delta_{\chi} \Phi\right] \simeq \partial_{\nu} \mathcal{Q}_{\mathrm{LW}}^{\mu \nu}[\Phi, \delta \Phi ; \chi]
$$

with

$$
\begin{aligned}
\mathcal{Q}_{\mathrm{LW}}^{\mu \nu}[\Phi, \delta \Phi ; \chi]= & \frac{1}{16 \pi}\left\{\delta K_{\mathrm{N}}^{\mu \nu}[\Phi ; \chi]-\delta K_{\mathrm{N}}^{\mu \nu}[\Phi ; \delta \chi]\right. \\
& \left.+2 \xi^{\mu \mu} \Theta^{\nu]}[\Phi, \delta \Phi]\right\} .
\end{aligned}
$$

In EMD theory the explicit form of $\mathcal{Q}_{\mathrm{LW}}^{\mu \nu}$ can be found by substituting Eqs. (25) and (3) into the above equation,

$$
\begin{aligned}
& \mathcal{Q}_{\mathrm{LW}}^{\mu \nu}[\Phi, \delta \Phi ; \chi] \\
& =\frac{\sqrt{-g}}{8 \pi}\left\{h^{\lambda[\mu} \nabla_{\lambda} \xi^{\nu]}-\xi^{\lambda} \nabla^{[\mu} h_{\lambda}^{\nu]}-\frac{1}{2} h \nabla^{[\mu} \xi^{\nu]}+\xi^{[\mu} \nabla_{\lambda} h^{\nu] \lambda}\right. \\
& -\xi^{[\mu} \nabla^{\nu]} h-\frac{1}{2}\left[\mathcal{N}\left(\delta F^{\mu \nu}+\frac{1}{2} h F^{\mu \nu}\right)+\mathcal{N}^{\prime} F^{\mu \nu} \delta \varphi\right]\left(\lambda+A_{\alpha} \xi^{\alpha}\right) \\
& \left.-\frac{1}{2} \mathcal{N} F^{\mu \nu} \xi^{\alpha} \delta A_{\alpha}-\mathcal{N} \xi^{[\mu} F^{\nu] \alpha} \delta A_{\alpha}-\xi^{[\mu} \partial^{\nu]} \varphi \delta \varphi\right\}
\end{aligned}
$$

where $h_{\mu \nu}=\delta g_{\mu \nu}$. We can use Eq. (29) and Stokes' theorem to write the conserved charge perturbation (20) as

$$
\delta Q_{\chi}=\oint_{\mathcal{D}} \mathcal{Q}_{\mathrm{LW}}^{\mu \nu}[\Phi, \delta \Phi ; \chi] d^{2} x_{\mu \nu}
$$

where $\mathcal{D}$ denotes the boundary of $\mathcal{C}$ and it is a spacelike codimension-2 surface. Usually it is thought that the linearization is only valid at spatial infinity. To overcome this problem, we integrate Eq. (32) over a one-parameter path in the solution phase space. To this end, suppose that $\Phi(\mathcal{W})$ is a collection of fields that solve the equations of motion of the EMD theory, where $\mathcal{W}$ is a free parameter in the solution phase space. Now, we replace $\mathcal{W}$ by $s \mathcal{W}$, where $0 \leq s \leq 1$ is just a parameter. By expanding $\Phi(s \mathcal{W})$ in terms of $s$, we have $\Phi(s \mathcal{W})=\Phi(0)+\left.s \frac{\partial \Phi}{\partial s}\right|_{s=0}+\cdots$. By substituting $\Phi=\Phi(s \mathcal{W})$ and $\delta \Phi=\left.d s \frac{\partial \Phi}{\partial s}\right|_{s=0}$ into Eq. (32), we can define the conserved charge conjugate to $\chi$. Then we have

$$
Q_{\chi}=\int_{0}^{1} d s \oint_{\mathcal{D}} \mathcal{Q}_{\mathrm{LW}}^{\mu \nu}[\Phi ; \chi \mid s] d^{2} x_{\mu \nu},
$$

where the integration over $s$ denotes integration over the one-parameter path in the solution phase space. In Eq. (33), $s=0$ is the value of the parameter corresponding to the background configuration. In this way, the background contribution in the conserved charge is subtracted, and thus the conserved charge will be always finite. Therefore, this method is applicable to spacetimes with any background. 


\section{NEAR-HORIZON FALL-OFF CONDITIONS AND SYMMETRIES}

Let us consider the near-horizon behavior of a stationary black hole in EMD theory. One can write the near-horizon metric in the Gaussian null coordinate system as [6,13-15]

$$
\begin{aligned}
d s^{2}= & -2 \kappa \rho d v^{2}+2 d v d \rho+2 \rho \theta_{A} d v d x^{A} \\
& +\left(\Omega_{A B}+\rho \lambda_{A B}\right) d x^{A} d x^{B}+\mathcal{O}\left(\rho^{2}\right),
\end{aligned}
$$

where $v$ is the advanced time coordinate such that a null surface is defined by $g^{\alpha \beta} \partial_{\alpha} v \partial_{\beta} v=0$ and the vector tangent to this surface is given by $k^{\mu}=g^{\mu \nu} \partial_{\nu} v$, which defines a ray. Also, $\rho$ is the affine parameter of the generator $k^{\mu}$. We assume that the horizon is located at $\rho=0$. Suppose $\kappa, \theta_{A}$, $\Omega_{A B}$, and $\lambda_{A B}$ are functions of $x^{A}$, where two coordinates $x^{A}$ are chosen constant along each ray. One can introduce the near-horizon fall-off conditions for the $U(1)$ gauge field and scalar field,

$$
\begin{gathered}
A_{v}=A_{v}^{(0)}+A_{v}^{(1)} \rho+\mathcal{O}\left(\rho^{2}\right), \quad A_{\rho}=0, \\
A_{B}=A_{B}^{(0)}+A_{B}^{(1)} \rho+\mathcal{O}\left(\rho^{2}\right), \\
\varphi=\varphi^{(0)}+\varphi^{(1)} \rho+\mathcal{O}\left(\rho^{2}\right),
\end{gathered}
$$

respectively, where we set $A_{\rho}=0$ as a gauge condition and $A_{v}^{(i)}, A_{B}^{(i)}$, and $\varphi^{(i)}$ are functions of $x^{A}$. By substituting the fall-off conditions (34), (35), and (36) into the field equations, we can find additional restrictions. The $(v, v)$ and $(v, A)$ components of the equations of motion (4) at zeroth order restrict $\kappa$ and $A_{v}^{(0)}$ to be constants, i.e., they are independent of $x^{A}$. The other components of the equations of motion relate first-order terms to zeroth-order ones in the metric, gauge field, and scalar field expansions, and we will not need them later.

In order to obtain the asymptotic symmetry generator $\chi$, we assume that the leading terms do not depend on the dynamical fields. Under such an assumption the boundary conditions will be "state independent," which means that the forms of the asymptotic symmetry generators are not considered to depend explicitly on the charges [14]. In this way we can find the components of the symmetry generator $\chi$ as follows [6,14]:

$$
\begin{aligned}
& \xi^{v}=T, \quad \xi^{\rho}=\frac{1}{2} \rho^{2} \Omega^{A B} \theta_{A} \partial_{B} T+\mathcal{O}\left(\rho^{3}\right), \\
& \xi^{A}=Y^{A}-\rho \Omega^{A B} \partial_{B} T+\frac{1}{2} \rho^{2} \Omega^{A C} \Omega^{B D} \lambda_{C D} \partial_{B} T+\mathcal{O}\left(\rho^{3}\right),
\end{aligned}
$$

$$
\begin{aligned}
\lambda= & \lambda^{(0)}+\rho \Omega^{A B} \varphi_{A} \partial_{B} T-\frac{1}{2} \rho^{2}\left(\Omega^{A C} \Omega^{B D} \lambda_{C D} \varphi_{A} \partial_{B} T\right. \\
& \left.-\Omega^{A B} \psi_{A} \partial_{B} T\right)+\mathcal{O}\left(\rho^{3}\right) .
\end{aligned}
$$

The symmetry generator $\chi$, with the above components, preserves the given near-horizon fall-off conditions. Here $T, Y^{A}$, and $\lambda^{(0)}$ are arbitrary functions of $x^{A}$. The change in the dynamical fields under the action of the symmetry generator $\chi$ can be read as

$$
\begin{gathered}
\delta_{\chi} \theta_{A}=\mathcal{L}_{Y} \theta_{A}-2 \kappa \partial_{A} T, \quad \delta_{\chi} \Omega_{A B}=\mathcal{L}_{Y} \Omega_{A B}, \\
\delta_{\chi} \lambda_{A B}=\mathcal{L}_{Y} \lambda_{A B}+\theta_{A} \partial_{B} T+\theta_{B} \partial_{A} T-2 \bar{\nabla}_{A} \bar{\nabla}_{B} T \\
\delta_{\chi} A_{v}^{(1)}=\mathcal{L}_{Y} A_{v}^{(1)}, \quad \delta_{\chi} A_{A}^{(0)}=\mathcal{L}_{Y} A_{A}^{(0)}+A_{v}^{(0)} \partial_{A} T+\partial_{A} \lambda^{(0)}, \\
\delta_{\chi} A_{A}^{(1)}=\mathcal{L}_{Y} A_{A}^{(1)}+A_{v}^{(1)} \partial_{A} T+\Omega^{B C}\left(\partial_{A} A_{B}^{(0)}-\partial_{B} A_{A}^{(0)}\right) \partial_{C} T \\
\delta_{\chi} \varphi^{(0)}=\mathcal{L}_{Y} \varphi^{(0)},
\end{gathered}
$$

where $\mathcal{L}_{Y}$ denotes the Lie derivative along $Y^{A}$ and $\bar{\nabla}_{A}$ is the covariant derivative with respect to the connection $\bar{\Gamma}_{B C}^{A}$ compatible with the metric of the horizon $\Omega_{A B}$. It is worth mentioning that because $\kappa$ and $A_{v}^{(0)}$ are not dynamical they will remain unchanged under the action of the symmetry generator $\chi$, i.e., $\delta_{\chi} \kappa=0$ and $\delta_{\chi} A_{v}^{(0)}=0$.

The asymptotic Killing vectors (37) are functions of the dynamical fields. To take this into account we introduce a modified version of Lie brackets [16],

$$
\left[\xi_{1}, \xi_{2}\right]=\mathcal{L}_{\xi_{1}} \xi_{2}-\delta_{\xi_{1}}^{(g)} \xi_{2}+\delta_{\xi_{2}}^{(g)} \xi_{1},
$$

where $\xi_{1}=\xi\left(T_{1}, Y_{1}^{A}\right)$ and $\xi_{2}=\xi\left(T_{2}, Y_{2}^{A}\right)$, and $\delta_{\xi_{1}}^{(g)} \xi_{2}$ denotes the change induced in $\xi_{2}$ due to the variation of the metric $\delta_{\xi_{1}} g_{\mu \nu}=\mathcal{L}_{\xi_{1}} g_{\mu \nu}$. Therefore, one finds that

$$
\left[\xi_{1}, \xi_{2}\right]=\xi_{12},
$$

with $\xi_{12}=\xi\left(T_{12}, Y_{12}^{A}\right)$, where

$T_{12}=Y_{1}^{A} \partial_{A} T_{2}-Y_{2}^{A} \partial_{A} T_{1}, \quad Y_{12}^{A}=Y_{1}^{B} \partial_{B} Y_{2}^{A}-Y_{2}^{B} \partial_{B} Y_{1}^{A}$.

Thus, the algebra of asymptotic Killing vectors is closed. In addition to $T$ and $Y^{A}$, the symmetry generator $\chi=$ $\chi\left(T, Y^{A}, \lambda^{(0)}\right)$ contains another degree of freedom, $\lambda^{(0)}$. Here, $\lambda^{(0)}$ is an arbitrary function of $x^{A}$ and generates $U(1)$ symmetry. Hence, we need to introduce two other commutators [6],

$$
\begin{aligned}
{\left[\chi\left(0,0,0, \lambda_{1}^{(0)}\right), \chi\left(0,0,0, \lambda_{2}^{(0)}\right)\right]=0 } \\
{\left[\chi\left(0,0,0, \lambda_{1}^{(0)}\right), \chi\left(0, Y_{2}^{A}, 0\right)\right]=-\left[\chi\left(0, Y_{2}^{A}, 0\right), \chi\left(0,0,0, \lambda_{1}^{(0)}\right)\right] } \\
=\chi\left(0,0,0,-\mathcal{L}_{Y_{2}} \lambda_{1}^{(0)}\right)
\end{aligned}
$$


in addition to Eq. (43). Equation (45) comes from the fact that $U(1)$ is an Abelian group, and we will justify Eq. (46) when we consider the algebra among conserved charges. In a nutshell, the algebra among the asymptotic symmetry generators can be written as

$$
\left[\chi_{1}, \chi_{2}\right]=\chi_{12},
$$

where $T_{12}$ and $Y_{12}^{A}$ are given by Eq. (44) and

$$
\lambda_{12}^{(0)}=\mathcal{L}_{Y_{1}} \lambda_{2}^{(0)}-\mathcal{L}_{Y_{2}} \lambda_{1}^{(0)} .
$$

Suppose the induced metric on the horizon $\Omega_{A B}$ is conformally related to an off-diagonal one $\gamma_{A B}$, i.e.,

$$
\Omega_{A B} d x^{A} d x^{B}=\Omega \gamma_{z \bar{z}} d z d \bar{z}
$$

where $\bar{z}$ is complex conjugate to $z$. For Kerr-Newman (A)dS black holes, $\gamma_{A B}$ describes the Riemann sphere. In this way, the Laurent expansion on the horizon is allowed. The general solution of the conformal Killing equations is $Y=Y^{z}(z) \partial_{z}+Y^{\bar{z}}(\bar{z}) \partial_{\bar{z}}$, and $T=T(z, \bar{z})$ and $\lambda^{(0)}=$ $\lambda^{(0)}(z, \bar{z})$ are arbitrary functions of $z$ and $\bar{z}$. Thus, we can define the modes as

$$
\begin{aligned}
T_{(m, n)} & =\chi\left(z^{m} \bar{z}^{n}, 0,0,0\right), \quad Y_{m}=\chi\left(0,-z^{m+1}, 0,0\right), \\
\bar{Y}_{m} & =\chi\left(0,0,-\bar{z}^{m+1}, 0\right), \quad \lambda_{(m, n)}^{(0)}=\chi\left(0,0,0, z^{m} \bar{z}^{n}\right),
\end{aligned}
$$

where $m, n \in \mathbb{Z}$. By using Eq. (47), we find that the algebra among these modes is given by

$$
\begin{aligned}
{\left[Y_{m}, Y_{n}\right] } & =(m-n) Y_{m+n}, \quad\left[\bar{Y}_{m}, \bar{Y}_{n}\right]=(m-n) \bar{Y}_{m+n}, \\
{\left[Y_{m}, \bar{Y}_{n}\right] } & =0, \quad\left[T_{(m, n)}, T_{(k, l)}\right]=0, \\
{\left[Y_{k}, T_{(m, n)}\right] } & =-m T_{(m+k, n)}, \quad\left[\bar{Y}_{k}, T_{(m, n)}\right]=-n T_{(m, n+k)},
\end{aligned}
$$

$$
\begin{aligned}
{\left[\lambda_{(m, n)}^{(0)}, \lambda_{(k, l)}^{(0)}\right] } & =0, \quad\left[Y_{k}, \lambda_{(m, n)}^{(0)}\right]=-m \lambda_{(m+k, n)}^{(0)}, \\
{\left[\bar{Y}_{k}, \lambda_{(m, n)}^{(0)}\right] } & =-n \lambda_{(m, n+k)}^{(0)}, \quad\left[\lambda_{(m, n)}^{(0)}, T_{(k, l)}\right]=0 .
\end{aligned}
$$

This algebra contains a set of supertranslation currents $T_{(m, n)}$ and two sets of Witt algebra currents, given by $Y_{m}$ and $\bar{Y}_{m}$. It also contains a set of multiple-charge currents $\lambda_{(m, n)}^{(0)}$. Two sets of Witt currents are in semi-direct sum with the supertranslation and multiple-charge currents. The subalgebra (51) is known as $\mathfrak{b}_{\mathfrak{g}} \mathfrak{g}_{4}^{H}$ [14] and differs from the Bondi-Metzner-Sachs algebra $\mathfrak{b}_{\mathfrak{m}} \mathfrak{g}_{4}$ [16-18] (the structure constants are different).

\section{NEAR-HORIZON CHARGES}

Now we find the conserved charge conjugate to the asymptotic symmetry generator $\chi$ obtained in the previous section. We take the codimension- 2 surface $\mathcal{D}$ in Eq. (33) to be the horizon,

$$
\begin{aligned}
\delta Q_{\chi} & =\oint_{H} \mathcal{Q}_{\mathrm{LW}}^{\mu \nu}[\Phi, \delta \Phi ; \chi] d^{2} x_{\mu \nu} \\
& =\left.\int d^{2} x \mathcal{Q}_{\mathrm{LW}}^{v \rho}\right|_{\rho=0} .
\end{aligned}
$$

By substituting the boundary conditions and components of the asymptotic symmetry generators into Eq. (53), we have

$$
\begin{aligned}
Q_{\chi}= & \frac{1}{8 \pi} \int d^{2} x \sqrt{\operatorname{det} \Omega}\left\{\left(\kappa-\frac{1}{2} \mathcal{N}^{(0)} A_{v}^{(0)} A_{v}^{(1)}\right) T\right. \\
& \left.-\frac{1}{2} Y^{A}\left(\theta_{A}+\mathcal{N}^{(0)} A_{v}^{(1)} A_{A}^{(0)}\right)-\frac{1}{2} \mathcal{N}^{(0)} A_{v}^{(1)} \lambda^{(0)}\right\},
\end{aligned}
$$

where an integral over a one-parameter path in solution phase space was taken. As we mentioned earlier, one can use Eqs. (21) and (22) to find the algebra among the conserved charges. After performing some calculations, we find that

$$
\left\{Q_{\chi_{1}}, Q_{\chi_{2}}\right\}=Q_{\left[\chi_{1}, \chi_{2}\right]}
$$

where $\left[\chi_{1}, \chi_{2}\right]$ is given by Eq. (47). In this case, by comparing Eqs. (21) and (55) we see that the central extension term does not appear. Since the algebra among the conserved charges is isomorphic to the algebra among the symmetry generators and the commutation relation (47) appears on the right-hand side of Eq. (55), it seems reasonable to consider such a commutation relation. By substituting Eq. (50) into Eq. (54), the supertranslation, superrotation, and multiple-charge modes can be obtained as

$$
\begin{gathered}
\mathcal{T}_{(m, n)}=\frac{1}{8 \pi} \int d z d \bar{z} \Omega \sqrt{\gamma}\left(\kappa-\frac{1}{2} \mathcal{N}^{(0)} A_{v}^{(0)} A_{v}^{(1)}\right) z^{m} \bar{z}^{n}, \\
\mathcal{Y}_{m}=\frac{1}{16 \pi} \int d z d \bar{z} \Omega \sqrt{\gamma}\left(\theta_{z}+\mathcal{N}^{(0)} A_{v}^{(1)} A_{z}^{(0)}\right) z^{m+1}, \\
\overline{\mathcal{Y}}_{m}=\frac{1}{16 \pi} \int d z d \bar{z} \Omega \sqrt{\gamma}\left(\theta_{\bar{z}}+\mathcal{N}^{(0)} A_{v}^{(1)} A_{\bar{z}}^{(0)}\right) \bar{z}^{m+1}, \\
\mathcal{Q}_{(m, n)}=-\frac{1}{16 \pi} \int d z d \bar{z} \Omega \sqrt{\gamma} \mathcal{N}^{(0)} A_{v}^{(1)} z^{m} \bar{z}^{n},
\end{gathered}
$$


respectively, where $\gamma=\operatorname{det}\left(\gamma_{A B}\right)$. By comparing the above equations with Eqs. (6.4), (6.7), (6.25), and (6.26) in Ref. [6], we deduce that Eqs. (57) and (59) will give us the correct value for the superrotation charges and multiple charges, respectively, but Eq. (56) will not give the correct value for the supertranslation charges. In fact, we expect that the supertranslation double-zeromode charge $\mathcal{T}_{(0,0)}$ will give us the black hole entropy multiplied by the Hawking temperature $T_{H}=\kappa / 2 \pi$.

Now, we deviate slightly from the discussion to express the difference between the results obtained in this paper and those in Ref. [6]. We compare conserved charge density expression (31) with that used in Ref. [6] [see Eq. (2.29)] where the scalar field was suppressed. They are different! The difference comes from the fact that in order to obtain Eq. (2.29) in Ref. [6] the gauge parameter $\lambda$ was redefined as $\lambda+\xi^{\mu} A_{\mu} \rightarrow \lambda$ (see Refs. [19] for a detailed discussion). This is the origin of the difference.

Now let us return to our discussion. Equation (55) gives us the algebra among charge modes,

$$
\begin{aligned}
\left\{\mathcal{Y}_{m}, \mathcal{Y}_{n}\right\} & =(m-n) \mathcal{Y}_{m+n}, \quad\left\{\overline{\mathcal{Y}}_{m}, \overline{\mathcal{Y}}_{n}\right\}=(m-n) \overline{\mathcal{Y}}_{m+n}, \\
\left\{\mathcal{Y}_{m}, \overline{\mathcal{Y}}_{n}\right\} & =0, \quad\left\{\mathcal{T}_{(m, n)}, \mathcal{T}_{(k, l)}\right\}=0, \\
\left\{\mathcal{Y}_{k}, \mathcal{T}_{(m, n)}\right\} & =-m \mathcal{T}_{(m+k, n)}, \quad\left\{\overline{\mathcal{Y}}_{k}, \mathcal{T}_{(m, n)}\right\}=-n \mathcal{T}_{(m, n+k)},
\end{aligned}
$$

$$
\begin{aligned}
\left\{\mathcal{Q}_{(m, n)}, \mathcal{Q}_{(k, l)}\right\} & =0, \quad\left\{\mathcal{Y}_{k}, \mathcal{Q}_{(m, n)}\right\}=-m \mathcal{Q}_{(m+k, n)}, \\
\left\{\overline{\mathcal{Y}}_{k}, \mathcal{Q}_{(m, n)}\right\} & =-n \mathcal{Q}_{(m, n+k)}, \quad\left\{\mathcal{Q}_{(m, n)}, \mathcal{T}_{(k, l)}\right\}=0
\end{aligned}
$$

In order to find the correct form of the supertranslation charges, first we consider the algebra among the supertranslation modes and multiple-charge modes [see Eq. (52)]. It is clear that $\lambda_{(m, n)}^{(0)}$ and $T_{(k, l)}$ commute with each other. By introducing the new mode

$$
\eta_{(m, n)}=\left(0,0,0, z^{m} \bar{z}^{n}\right),
$$

we construct a subalgebra of the algebra (52). Therefore, we can define new supertranslation modes as

$$
T_{(m, n)}^{(\text {new })}=T_{(m, n)}+\eta_{(m, n)},
$$

so that they obey the same algebra that the old ones do. Strictly speaking, these new modes obey the algebra (51) and (52) with $T_{(m, n)} \rightarrow T_{(m, n)}^{(\text {new })}$. Thus, we are allowed to use $U(1)$ gauge fixing to find the correct form of supertranslation charges. To this end, we fix the $U(1)$ gauge freedom as

$$
\lambda^{(0)}=-A_{v}^{(0)} T,
$$

so that corresponding modes are given by Eq. (62). In this way, we can define new supertranslation charges conjugate to the supertranslation modes $T_{(m, n)}^{(\text {new })}$ as

$$
\mathcal{T}_{(m, n)}^{(\text {new })}=\frac{\kappa}{8 \pi} \int d z d \bar{z} \Omega \sqrt{\gamma} z^{m} \bar{z}^{n}
$$

This is exactly what we were looking for. In fact, these are charge modes corresponding to charges conjugate to the symmetry generator $\chi=\chi\left(T, 0,0,-A_{v}^{(0)} T\right)$. Eventually, we perform a redefinition as $\tilde{\mathcal{T}}_{(m, n)}=\frac{1}{2 \kappa} \mathcal{T}_{(m, n)}^{(\text {new })}$. In this way, we expect that the supertranslation double-zero mode $\tilde{\mathcal{T}}_{(0,0)}$ can be related to the black hole entropy as $[6,14,15]$

$$
\mathcal{S}=4 \pi \tilde{\mathcal{T}}_{(0,0)}
$$

Since $\lambda^{(0)}$ is in general a dynamical field-independent function and we set it as Eq. (64) in the last step, i.e., when we want to calculate charges, then $\tilde{\mathcal{T}}_{(0,0)}$ will satisfy the same algebra as Eqs. (60) and (61). Now, we replace the brackets by commutators, namely, $\{,\} \equiv i[$,$] ; then, the algebra$ among charge modes becomes

$$
\begin{aligned}
i\left[\mathcal{Y}_{m}, \mathcal{Y}_{n}\right] & =(m-n) \mathcal{Y}_{m+n}, & & i\left[\overline{\mathcal{Y}}_{m}, \overline{\mathcal{Y}}_{n}\right]=(m-n) \overline{\mathcal{Y}}_{m+n}, \\
i\left[\mathcal{Y}_{k}, \tilde{\mathcal{T}}_{(m, n)}\right] & =-m \tilde{\mathcal{T}}_{(m+k, n)}, & & i\left[\overline{\mathcal{Y}}_{k}, \tilde{\mathcal{T}}_{(m, n)}\right]=-n \tilde{\mathcal{T}}_{(m, n+k)}, \\
i\left[\mathcal{Y}_{k}, \mathcal{Q}_{(m, n)}\right] & =-m \mathcal{Q}_{(m+k, n)}, & & i\left[\overline{\mathcal{Y}}_{k}, \mathcal{Q}_{(m, n)}\right]=-n \mathcal{Q}_{(m, n+k)},
\end{aligned}
$$

where commutators not displayed vanish.

It is expected that we can apply the Sugawara deconstruction proposed in Ref. [5]. To do this, we introduce four new generators $\hat{J}_{m}^{ \pm}$and $\hat{K}_{m}^{ \pm}$so that they obey the algebra

$$
i\left[\hat{J}_{m}^{ \pm}, \hat{K}_{n}^{ \pm}\right]=m \delta_{m+n, 0},
$$

where commutators not displayed vanish. The algebra (68) consists of two copies of the three-dimensional flat-space near-horizon symmetry algebra [20]. Hence, we can construct generators $\tilde{\mathcal{T}}_{(m, n)}, \mathcal{Y}_{m}^{(\text {new })}$, and $\overline{\mathcal{Y}}_{m}^{(\text {new })}$ as follows:

$$
\begin{aligned}
& \tilde{\mathcal{T}}_{(m, n)}=\hat{J}_{m}^{+} \hat{J}_{n}^{-}, \quad \mathcal{Y}_{m}^{(\text {new })}=\sum_{p} \hat{J}_{m-p}^{+} \hat{K}_{p}^{+}, \\
& \overline{\mathcal{Y}}_{m}^{\text {(new) }}=\sum_{p} \hat{J}_{m-p}^{-} \hat{K}_{p}^{-} .
\end{aligned}
$$

It is easy to check that the definitions presented in Eq. (69) obey the algebra (67) provided that $\hat{J}_{m}^{ \pm}$and $\hat{K}_{m}^{ \pm}$satisfy the algebra introduced in Eq. (68). There will be six algebraic constraints on charge zero modes (because we assume that charge zero modes are complex numbers),

$$
\frac{\mathcal{S}}{4 \pi}=\hat{J}_{0}^{+} \hat{J}_{0}^{-}, \quad \pm \frac{i}{2} \mathcal{J}=\hat{J}_{0}^{ \pm} \hat{K}_{0}^{ \pm},
$$

where $\mathcal{J}$ is the angular momentum of a black hole. 


\section{EXAMPLES}

Let us consider a typical static dilaton black hole $[1,21,22]$ as a first example. The procedure performed in Secs. III and IV is independent of whether the black object is a black hole or a black string, etc. Therefore, as a second example, we consider a black string solution of EMD theory. In both examples we set $\Lambda=0$ and $\mathcal{V}=0$.

\section{A. Static dilaton black hole}

Consider a typical static dilaton black hole

$$
\begin{aligned}
d s^{2} & =-F(r) d v^{2}+2 d v d r+H(r)\left(d \theta^{2}+\sin ^{2} \theta d \phi^{2}\right), \\
\varphi & =\varphi_{\infty}+\ln \left|\frac{r+\Sigma}{r-\Sigma}\right|, \quad A=\left(\frac{e^{\frac{1}{2} \varphi_{\infty}} q}{r-\Sigma}\right) d v,
\end{aligned}
$$

with

$$
\begin{aligned}
& F(r)=\frac{(r-2 M-\Sigma)(r+\Sigma)}{r^{2}-\Sigma^{2}}, \\
& H(r)=r^{2}-\Sigma^{2}, \quad \Sigma=-\frac{q^{2}}{2 M},
\end{aligned}
$$

where $0 \leq r<\infty, 0 \leq \theta \leq \pi$, and $0 \leq \phi<2 \pi$ are the radial, polar, and azimuthal coordinates, respectively. This black hole has three parameters $M, q$, and $\varphi_{\infty}$ and solves the equations of motion (4)-(6) with $\mathcal{N}(\varphi)=$ $4 \exp (-\varphi)(\Lambda=0$ and $\mathcal{V}=0$ were assumed $)$. The event horizon is located at $r_{H}=2 M+\Sigma$. Now, we define a new radial coordinate $\rho=r-r_{H}$. We can expand the metric, $U(1)$ gauge field, and scalar field with respect to $\rho$ and find that the corresponding fall-off conditions can be written as Eqs. (34)-(36), where the explicit form of $\kappa, A_{v}^{(0)}$, and the dynamical fields are given as

$$
\begin{aligned}
\kappa & =\frac{1}{4 M}, \quad \theta_{A}=0, \quad \Omega_{\theta \theta}=\left(4 M^{2}-2 q^{2}\right), \\
\Omega_{\phi \phi} & =\left(4 M^{2}-2 q^{2}\right) \sin ^{2} \theta, \quad \Omega_{\theta \phi}=0, \\
A_{v}^{(0)} & =\frac{e^{\frac{1}{2} \varphi_{\infty} q}}{2 M}, \quad A_{v}^{(1)}=-\frac{e^{\frac{1}{2} \varphi_{\infty}} q}{4 M^{2}}, \\
A_{A}^{(0)} & =A_{A}^{(1)}=0, \quad \varphi^{(0)}=\varphi_{\infty}+\ln \left|\frac{2 M^{2}-q^{2}}{2 M^{2}}\right| .
\end{aligned}
$$

The full 2-metric on the horizon,

$$
d \sigma^{2}=\left(4 M^{2}-2 q^{2}\right)\left[d \theta^{2}+\sin ^{2} \theta d \phi^{2}\right],
$$

is conformally related to the Riemann sphere. To show this relation, one can introduce a change of coordinates $z=$ $e^{i \phi} \cot \theta / 2$ and $\bar{z}=e^{-i \phi} \cot \theta / 2$ and show that the conformal factor is given by $\Omega=\left(4 M^{2}-2 q^{2}\right)$.

Now we can calculate the supertranslation double-zero mode $\tilde{\mathcal{T}}_{(0,0)}=\frac{1}{4}\left(4 M^{2}-2 q^{2}\right)$. Therefore, the static dilaton black hole entropy is

$$
\mathcal{S}=\pi\left(4 M^{2}-2 q^{2}\right),
$$

where Eq. (66) was used. This result is consistent with the previous result obtained in Ref. [23]. It is clear that the superrotation charge modes $\mathcal{Y}_{m}$ and $\overline{\mathcal{Y}}_{m}$ are zero. By substituting Eqs. (73) and (74) into Eq. (59), one can find the multiple-charge double-zero mode as

$$
\mathcal{Q}_{(0,0)}=q e^{-\frac{1}{2} \varphi_{\infty}},
$$

which is exactly the total electric charge of a static dilaton black hole [23].

The given black hole has inner and outer horizons of radius $r_{-}=-\Sigma$ and $r_{+}=2 M+\Sigma$, respectively. One can choose the zero-mode eigenvalues of $\hat{J}_{m}^{ \pm}$and $\hat{K}_{m}^{ \pm}$as

$$
\hat{J}_{0}^{ \pm}=\frac{1}{2}\left(r_{+} \pm i r_{-}\right), \quad \hat{K}_{0}^{ \pm}=0
$$

so that they satisfy Eq. (70).

\section{B. Rotating charged dilaton black string}

Now consider a rotating charged dilaton black string [3]

$$
\begin{aligned}
d s^{2}= & -F(r)(\Xi d t-a d \hat{\phi})^{2}+\frac{d r^{2}}{F(r)}+R(r)\left(\frac{a}{l^{2}} d t-\Xi d \hat{\phi}\right)^{2} \\
+ & R(r) \frac{d x^{2}}{l^{2}}, \\
& \varphi=\frac{\beta}{\alpha} \ln \left(\frac{b}{r}\right), \quad A=-\frac{q}{r}(\Xi d t-a d \hat{\phi}),
\end{aligned}
$$

with

$$
\begin{aligned}
F(r) & =r^{\beta-1}\left(-M+\frac{\left(1+\alpha^{2}\right) q^{2}}{b^{\beta} r}\right), \quad R(r)=b^{\beta} r^{2-\beta}, \\
\Xi^{2} & =1+\frac{a^{2}}{l^{2}}, \quad \beta=\frac{2 \alpha^{2}}{1+\alpha^{2}},
\end{aligned}
$$

where $-\infty<t<\infty$ is the time coordinate. The line element (78) describes a black string for $0 \leq \hat{\phi}<2 \pi$ and $-\infty<x<\infty$. This black string has three parameters $M>0, q$, and $b$ and solves the equations of motion (4)-(6) with $\mathcal{N}(\varphi)=4 \exp (-\alpha \varphi)$, where $\alpha$ is a constant. Also, $l$ is a constant and has dimensions of length. The spacetime described by Eq. (78) presents a naked singularity with a regular cosmological horizon at

$$
r_{H}=\frac{\left(1+\alpha^{2}\right) q^{2}}{b^{\beta} M} .
$$

In order to find the near-horizon geometry of a rotating charged dilaton black string, first we write the metric (78) in the advanced Eddington-Finkelstein coordinates $(v, r, \tilde{\phi}, x)$. To this end, we transform the coordinates as 
$d v=d t+\frac{1-B(r) g_{t \hat{\phi}}}{g_{t t}} d r, \quad d \tilde{\phi}=d \hat{\phi}+B(r) d r$,

with

$$
B(r)=\frac{a}{F(r) R(r) l^{2}} \sqrt{R(r)\left[R(r)-l^{2} F(r)\right]} .
$$

Next, we perform another coordinate transformation as $\tilde{\phi}=\phi+\Omega_{H} v$, where $\Omega_{H}=\frac{a}{\Xi l^{2}}$ is the horizon angular velocity of a rotating charged dilaton black string. Finally, we find that

$$
\begin{aligned}
d s^{2}= & -\frac{F}{\Xi^{2}} d v^{2}+\frac{2 F\left(1+\frac{a}{\Xi l^{2}} R B\right)}{\left(F \Xi^{2}-\frac{a^{2}}{l^{4}} R\right)} d v d r+\frac{2 a F}{\Xi} d v d \phi \\
& +\frac{2 F R B\left(1+\frac{\Xi l^{2}}{a} F B\right)}{\left(F \Xi^{2}-\frac{a^{2}}{l^{4}} R\right)} d r d \phi \\
& +\left(-F a^{2}+R \Xi^{2}\right) d \phi^{2}+R \frac{d x^{2}}{l^{2}} .
\end{aligned}
$$

In this coordinate system the $U(1)$ gauge field can be written as

$$
A=-\frac{q}{r}\left(\frac{d v}{\Xi}-a d \phi\right)
$$

and the scalar field remains unchanged. Also, we have $g_{v v}=$ $g_{v \phi}=0$ on the cosmological horizon. Now we write the nearhorizon fall-off conditions for a rotating charged dilaton black string in the Gaussian null coordinate system. To do this, we follow the method proposed in Appendix A of Ref. [24]. Therefore, we rewrite the metric relative to the correct set of geodesics. A suitable pair of cross-normalized null normals is

$l=\partial_{v}$, and $n=\left(\frac{l^{4}(1+\Xi)^{2}}{2 a^{2} b^{\beta} r_{H}^{2-\beta}}\right) \partial_{v}-\Xi \partial_{r}-\left(\frac{l^{2}(1+\Xi)}{a b^{\beta} r_{H}^{2-\beta} \Xi}\right) \partial_{\phi}$.

These vectors are defined on the horizon and we have $l$. $\left.l\right|_{H}=\left.n \cdot n\right|_{H}=0$ and $l \cdot n=1$. Now we consider a family of null geodesics that cross $H$. The vector field tangent to them is $n$ and they are labeled by $(v, \theta, \phi)$. Suppose $\rho$ is an affine parameter which parametrizes the given geodesics so that $\rho=0$ on $H$. The geodesics can be constructed up to second order in $\rho$ :

$$
X_{(v, \theta, \phi)}^{\mu}(\rho)=\left.X^{\mu}\right|_{\rho=0}+\left.\rho \frac{d X^{\mu}}{d \rho}\right|_{\rho=0}+\mathcal{O}\left(\rho^{2}\right)
$$

where $\left.X^{\mu}\right|_{\rho=0}=\left(v, r_{H}, \theta, \phi\right)$ and $\left.\frac{d X^{\mu}}{d \rho}\right|_{\rho=0}=n^{\mu}$. Equation (87) defines a transformation from $(v, r, \theta, \phi)$ to $(v, \rho, \theta, \phi)$, and thus we can calculate the first-order expansion of the metric $g_{\mu \nu}=g_{\mu \nu}^{(0)}+\rho g_{\mu \nu}^{(1)}+\mathcal{O}\left(\rho^{2}\right)$, where

$$
\begin{gathered}
g_{v \rho}^{(0)}=1, \quad g_{\phi \phi}^{(0)}=b^{\beta} r_{H}^{2-\beta} \Xi^{2}, \quad g_{x x}^{(0)}=\frac{b^{\beta} r_{H}^{2-\beta}}{l^{2}}, \\
g_{v v}^{(1)}=-2 \kappa, \quad g_{v \phi}^{(1)}=a M r_{H}^{\beta-2},
\end{gathered}
$$

where $\kappa$ is the surface gravity of the rotating charged dilaton black string,

$$
\kappa=\frac{M r_{H}^{\beta-2}}{2 \Xi} .
$$

In the new coordinate system, the $U(1)$ gauge field and scalar field can be expanded as

$$
\begin{aligned}
A_{v} & =-\frac{q}{r_{H} \Xi}-\frac{q}{r_{H}^{2}} \rho+\mathcal{O}\left(\rho^{2}\right), \quad A_{\phi}=\frac{a q}{r_{H}}+\frac{a q \Xi}{r_{H}^{2}}+\mathcal{O}\left(\rho^{2}\right), \\
A_{\rho} & =\mathcal{O}\left(\rho^{2}\right), \quad A_{x}=\mathcal{O}\left(\rho^{2}\right), \\
\varphi & =\frac{\beta}{\alpha} \ln \left(\frac{b}{r_{H}}\right)+\frac{\beta \Xi}{\alpha r_{H}} \rho+\mathcal{O}\left(\rho^{2}\right) .
\end{aligned}
$$

From Eq. (88), the full 2-metric on the horizon is

$$
d \sigma^{2}=b^{\beta} r_{H}^{2-\beta}\left(\Xi^{2} d \phi^{2}+\frac{d x^{2}}{l^{2}}\right) .
$$

This metric is conformally related to a cylinder. To show this relation, we introduce the field-dependent change of coordinates

$$
z=\exp \left(\frac{x}{\Xi l}+i \phi\right), \quad \bar{z}=\exp \left(\frac{x}{\Xi l}-i \phi\right) .
$$

Now, we can write the metric of the horizon in the conformal form

$$
d \sigma^{2}=\Omega \gamma_{A B} d x^{A} d x^{B}=\Omega d z d \bar{z},
$$

with

$$
\Omega=b^{\beta} r_{H}^{2-\beta} \Xi^{2} \exp \left(-\frac{2 x}{\Xi l}\right) .
$$

Here, $\gamma_{A B}$ is the metric on a cylinder. The conformal factor $\Omega$ is a function of $z$ and $\bar{z}$. The induced metric on the horizon is locally, conformally equivalent to a cylinder and hence the Laurent expansion on the cylinder is allowed. Therefore, we can use Eqs. (65) and (57)-(59) to find the charge modes on the horizon of the rotating charged black string.

The supertranslation double-zero-mode charge per unit length of the string can be obtained as 


$$
\tilde{\mathcal{T}}_{(0,0)}=\frac{b^{\beta} r_{H}^{2-\beta} \Xi}{8 l}
$$

and hence the entropy per unit length of the string will be

$$
\mathcal{S}=\frac{\pi b^{\beta} r_{H}^{2-\beta} \Xi}{2 l}
$$

where Eq. (66) was used. Also, one can show that the superrotation charge and multiple-charge modes per unit length of the string are

$$
\begin{aligned}
\mathcal{Y}_{m} & =\frac{i a M \Xi b^{\beta}}{16 l}\left(\frac{3-\alpha^{2}}{1+\alpha^{2}}\right) \delta_{m, 0}, \\
\overline{\mathcal{Y}}_{m} & =-\frac{i a M \Xi b^{\beta}}{16 l}\left(\frac{3-\alpha^{2}}{1+\alpha^{2}}\right) \delta_{m, 0}, \\
\mathcal{Q}_{(0,0)} & =\frac{q \Xi}{2 l},
\end{aligned}
$$

respectively. Therefore, the angular momentum and electric charge per unit length of the string can be read as

$$
\mathcal{J}=\frac{a M \Xi b^{\beta}}{8 l}\left(\frac{3-\alpha^{2}}{1+\alpha^{2}}\right), \quad \mathcal{Q}_{E}=\frac{q \Xi}{2 l},
$$

respectively. One can choose the zero-mode eigenvalues of $\hat{J}_{m}^{ \pm}$and $\hat{K}_{m}^{ \pm}$as

$$
\begin{aligned}
\hat{J}_{0}^{ \pm} & =\left(\frac{b^{\beta} r_{H}^{2-\beta}}{8 l \Xi}\right)^{\frac{1}{2}}\left(1 \pm \frac{i a}{l}\right), \\
\hat{K}_{0}^{ \pm} & =-\left(\frac{b^{\beta} r_{H}^{2-\beta}}{8 l \Xi}\right)^{-\frac{1}{2}}\left(\frac{3-\alpha^{2}}{1+\alpha^{2}}\right)\left(\frac{a^{2} b^{\beta} M}{16 l^{2} \Xi}\right)\left(1 \pm \frac{i l}{a}\right),
\end{aligned}
$$

so that they satisfy Eq. (70). The results in Eqs. (97) and (99) agree with the results obtained in Ref. [3].

\section{CONCLUSION}

In this paper, we have considered EMD theory. The conserved charge conjugate to the symmetry generator $\chi=(\xi, \lambda)$ can be obtained using Eq. (31) in EMD theory. Equation (31) differs from the one obtained in Ref. [19] and that used in Ref. [6]. The difference comes from the fact that in obtaining Eq. (2.29) in Refs. [6,19], the gauge parameter $\lambda$ was redefined as $\lambda+\xi^{\mu} A_{\mu} \rightarrow \lambda$. In Sec. III, we considered the near-horizon fall-off conditions for the metric, $U(1)$ gauge field, and scalar field in the Gaussian null coordinate system. The equations of motion imply that the surface gravity $\kappa$ and first-order term of the timelike component of the $U(1)$ gauge field $A_{v}^{(0)}$ have to be constants for stationary black holes. The components of the symmetry generator $\chi$ are given by Eqs. (37) and (38). The changes in the dynamical fields under the action of the symmetry generator $\chi$ are given by Eqs. (39) and (40). We have assumed that the induced metric on the horizon $\Omega_{A B}$ is conformally related to an offdiagonal one $\gamma_{A B}$ (where $x^{A}=\{z, \bar{z}\}$ and $\bar{z}$ is complex conjugate to $z$ ), and hence the Laurent expansion on the horizon is allowed. The supertranslation, superrotation, and multiple-charge modes are given by Eq. (50) and the algebra among them contains a set of supertranslations currents $T_{(m, n)}$ and two sets of Witt algebra currents, given by $Y_{m}$ and $\bar{Y}_{m}$. It also contains a set of multiple-charge currents $\lambda_{(m, n)}^{(0)}$. Two sets of Witt currents are in semi-direct sum with the supertranslation and multiple-charge currents. We have obtained the near-horizon conserved charge conjugate to the near-horizon symmetry generator $\chi$ and shown that the algebra among the conserved charges is given by Eq. (55). Consequently, Eqs. (56)-(59) give us charge modes. It was expected that the supertranslation double-zero-mode charge $\mathcal{T}_{(0,0)}$ would give us the black hole entropy multiplied by Hawking temperature. Because of the presence of the second term in the integrand in Eq. (56), this statement was not the case. This may be seen as a problem, but it is not. Because EMD theory has an extra $U(1)$ degree freedom, we can use an appropriate gauge fixing to fix the problem. To this end, we introduced new supertranslation modes $T_{(m, n)}^{(\text {new })}$ [see Eq. (63)] and fixed the $U(1)$ gauge freedom as Eq. (64). The supertranslation charges (65) conjugate to these new supertranslation modes are exactly what we were looking for. By redefining the supertranslation charges as $\tilde{\mathcal{T}}_{(m, n)}=\frac{1}{2 \kappa} \mathcal{T}_{(m, n)}^{(\text {new })}$, the relation between the black hole entropy $\mathcal{S}$ and $\tilde{\mathcal{T}}_{(0,0)}$ is given by Eq. (66). We have shown that $\tilde{\mathcal{T}}_{(m, n)}$ together with the superrotation charge modes and multiple-charge modes satisfy the algebra (67). By introducing four new generators $\hat{J}_{m}^{ \pm}$and $\hat{K}_{m}^{ \pm}$so that they obey the algebra (68), we were able to apply the Sugawara deconstruction proposed in Ref. [5]. In this way, we were able to construct generators $\tilde{\mathcal{T}}_{(m, n)}, \mathcal{Y}_{m}^{(\text {new })}$, and $\overline{\mathcal{Y}}_{m}^{(\text {new) }}$ as in Eq. (69). Hence, the relation among the zero modes is given by Eq. (70), where $\mathcal{J}$ is the angular momentum of black hole. In Sec. V, we considered two examples: a typical static dilaton black hole and a charged rotating black string. Because the procedure performed in Secs. III and IV is independent of whether the black object is a black hole or a black string, we were able to apply the method to a black string as well as a black hole. In both examples, we assumed that $\Lambda=\mathcal{V}=0$. For a typical static dilaton black hole, the full 2-metric on the horizon is conformally related to the Riemann sphere. Using Eqs. (56)-(59), we found the charge zero modes. The results obtained are exactly match those in previous works. In Sec. V B, we wrote the near-horizon fall-off-conditions corresponding to a rotating charged dilaton black string in the Gaussian null coordinate system [see Eqs. (88)-(91)]. We have shown that the induced metric on the horizon of a rotating charged dilaton black string is locally conformally 
equivalent to a cylinder. Therefore, we used Eqs. (65) and (57)-(59) to find the value of the charge zero modes per unit length of the string. Using these two examples, we have shown that the new entropy formula proposed in Ref. [5] is valid in EMD theory and for black strings.

\section{ACKNOWLEDGMENTS}

The work of Hamed Adami has been financially supported by the Research Institute for Astronomy Astrophysics of Maragha (RIAAM) under research Project No. 1/5750-4.
[1] D. Garfinkle, G. T. Horowitz, and A. Strominger, Phys. Rev. D 43, 3140 (1991); 45, 3888(E) (1992).

[2] M. Mars and W. Simon, Adv. Theor. Math. Phys. 6, 279 (2002).

[3] M. H. Dehghani and N. Farhangkhah, Phys. Rev. D 71, 044008 (2005).

[4] A. Sheykhi, M. H. Dehghani, N. Riazi, and J. Pakravan, Phys. Rev. D 74, 084016 (2006).

[5] H. Gonzalez, D. Grumiller, W. Merbis, and R. Wutte, EPJ Web Conf. 168, 01009 (2018).

[6] M. R. Setare and H. Adami, J. High Energy Phys. 04 (2018) 133.

[7] J. Lee and R. M. Wald, J. Math. Phys. (N.Y.) 31, 725 (1990).

[8] R. M. Wald, Phys. Rev. D 48, R3427 (1993).

[9] V. Iyer and R. M. Wald, Phys. Rev. D 50, 846 (1994).

[10] K. Prabhu, Classical Quantum Gravity 34, 035011 (2017).

[11] K. Hajian and M. M. Sheikh-Jabbari, Phys. Rev. D 93, 044074 (2016).

[12] M. R. Setare and H. Adami, Classical Quantum Gravity 34, 105008 (2017).

[13] L. A. Tamburino and J. H. Winicour, Phys. Rev. 150, 1039 (1966).
[14] L. Donnay, G. Giribet, H. A. Gonzalez, and M. Pino, J. High Energy Phys. 09 (2016) 100.

[15] L. Donnay, G. Giribet, H. A. Gonzalez, and M. Pino, Phys. Rev. Lett. 116, 091101 (2016).

[16] G. Barnich and C. Troessaert, J. High Energy Phys. 05 (2010) 062.

[17] G. Barnich and C. Troessaert, Phys. Rev. Lett. 105, 111103 (2010).

[18] G. Barnich and C. Troessaert, J. High Energy Phys. 12 (2011) 105.

[19] S. Hyun, J. Jeong, S.-A. Park, and S.-H. Yi, Phys. Rev. D 90, 104016 (2014).

[20] H. Afshar, D. Grumiller, W. Merbis, A. Perez, D. Tempo, and R. Troncoso, Phys. Rev. D 95, 106005 (2017).

[21] J. Preskill, P. Schwarz, A. D. Shapere, S. Trivedi, and F. Wilczek, Mod. Phys. Lett. A 06, 2353 (1991).

[22] R. Kallosh, A. D. Linde, T. Ortin, A. W. Peet, and A. V. Proeyen, Phys. Rev. D 46, 5278 (1992).

[23] K. Hajian and M. M. Sheikh-Jabbari, Phys. Lett. B 768, 228 (2017).

[24] I. Booth, Phys. Rev. D 87, 024008 (2013). 